\title{
Phrases sans parole : À propos d'une histoire d'amour du $X X^{\mathrm{e}}$ siècle
}

\author{
Patron, Sylvie \\ Université Paris Diderot-Paris 7 \\ Centre d'études et de recherches interdisciplinaires de l'UFR Lettres, arts, cinéma (CÉRILAC- EA 4011) \\ sylvie.patron@orange.fr
}

\section{Préliminaires}

1.1 Cet article porte sur les relations entre récit (plus précisément narration) et fiction dans une nouvelle de Mario Benedetti, «Cinq ans de vie», extraite des Histoires de Paris ${ }^{1}$. Son cadre de référence théorique est la théorie non communicationnelle ou théorie poétique de la narration de S.-Y. Kuroda et Ann Banfield ${ }^{2}$. Sans m'attarder sur les divergences ponctuelles entre ces deux auteurs, je retiendrai de leurs travaux les six propositions suivantes :

(1) Les théories communicationnelles du récit, basées sur les concepts de narrateur et de narrataire, réels ou fictionnels, ne peuvent rendre compte que d'une sous-catégorie de récits de fiction : les récits de fiction à la première personne et même, selon Banfield, les récits de fiction à la première personne où le narrateur s'adresse explicitement à un narrataire et où, d'un point de vue formel, le récit est raconté comme communication.

(2) Il existe des récits de fiction avec narrateur (sous-entendu fictionnel) et des récits de fiction sans narrateur, ce qui ne veut pas dire que personne ne les a produits, mais simplement qu'ils ne contiennent aucune marque linguistique renvoyant à un sujet et à une situation d'énonciation, réels ou fictionnels. On peut parler d'effacement ou de retrait énonciatif de l'auteur du récit de fiction (pour Banfield, l'auteur «n'est pas directement incarné dans une première personne, comme l'est un locuteur dans son discours », « il ne parle pas [...], il écrit et, en écrivant, disparaît ${ }^{3}$ ). On doit parler d'absence de narrateur (et non d'effacement énonciatif du narrateur ou, comme on dit plus souvent, de «narrateur effacé ») lorsque le récit ne contient aucune marque linguistique renvoyant à un sujet et à une situation d'énonciation fictionnels. Les phrases du style indirect libre (à la troisième personne et au passé) en français ou en anglais constituent un cas d'observation singulier qui falsifie l'hypothèse du narrateur effacé.

(3) Le narrateur omniscient est, dans la plupart des cas, une explication ad hoc (dans la plupart des cas, car on ne peut négliger les cas où l'auteur, établissant une sorte de tourniquet entre l'invention et le savoir, se représente lui-même dans son récit en tant que narrateur omniscient). Comme l'écrit Kuroda, « [1]e narrateur omniscient ne peut être identifié par aucun mécanisme linguistique dont l'existence soit établie indépendamment de l'hypothèse qu'il existe un tel narrateur, alors que cette possibilité existe pour le narrateur des [récits à la première personne] $»^{4}$.

(4) Il convient de distinguer clairement ce qui relève du contenu de la représentation fictionnelle (les personnages et leurs paroles, pensées, états de conscience) et ce qui relève des moyens mobilisés au service de la construction de cette représentation (la langue, le style de la représentation). Banfield écrit à propos de la représentation de la conscience : «[Elle] n'est pas la "reproduction réaliste" de l'activité mentale; elle ne crée pas des "esprits transparents". L'esprit n'est jamais transparent, pas même au "narrateur omniscient". Son contenu fait l'objet de reconstructions et de représentations hypothétiques dans une langue [susceptible de refléter différents modes de conscience]. C'est l'a priori de la double voix qui conduit à avoir recours à la voix d'un narrateur pour rendre compte de l'écart entre le style de la représentation et celui que le personnage aurait employé s'il avait lui-même parlé ».5. 
(5) Le récit de fiction moderne à la troisième personne peut être considéré comme une collection structurée d'informations, les unes sans origine assignable dans le monde de la fiction (on peut parler de phrases ou de contextes narratifs, ou narratifs zéro, ou encore objectifs, par opposition à subjectifs), les autres provenant d'un ou de plusieurs sujets de conscience appartenant au monde de la fiction (on peut parler de phrases ou de contextes subjectifs). Je laisse de côté les phrases du discours direct, dont l'analyse n'est pas fondamentalement différente dans les théories communicationnelles et dans celles de Kuroda et Banfield. Les phrases narratives ou objectives ne peuvent pas être conçues comme la représentation d'un point de vue distinct de celui des personnages. Elles ne représentent aucun point de vue et ne peuvent pas être fausses. Elles établissent ce qui est de l'ordre des faits dans le monde de la fiction. Les autres phrases expriment une subjectivité, mais une subjectivité qui n'est pas couplée avec le syncrétisme traditionnel je-ici-maintenant (une «subjectivité de troisième personne », si l'on veut). Elles présentent les faits fictionnels selon le point de vue d'un ou de plusieurs personnages qui ne sont pas en position de locuteurs. Le recours à la notion de contexte s'impose dans la mesure où certaines phrases ne comportent pas d'éléments ou de constructions subjectifs (définis comme tels sur la base d'une analyse linguistique précise), mais apparaissent au milieu d'autres phrases comportant, elles, ces éléments ou constructions caractéristiques, et sont dès lors interprétées comme subjectives.

(6) La lecture d'un récit de fiction moderne à la troisième personne implique que l'on reconnaisse les intentions de l'auteur relativement au caractère objectif ou subjectif des phrases ou des contextes. En cela, il y a bien une forme de communication ou de co-intentionnalité entre l'auteur et le lecteur, mais Kuroda et Banfield préfèrent réserver le terme " communication » pour la communication entre un locuteur et un allocutaire proprement dits, c'est-à-dire un locuteur qui peut directement inscrire sa subjectivité dans l'énoncé et un allocutaire qui est celui visé par le locuteur, ce qui peut également être marqué dans l'énoncé.

Si j'ai choisi cette théorie en lieu et place de la narratologie, qui a constitué pour des générations un cadre de référence, c'est qu'elle me semble supérieure à cette dernière sur plusieurs points. Tout d'abord, elle explique pourquoi l'auteur choisit la forme du récit de fiction à la première ou à la troisième personne pour présenter l'histoire. Elle évite la réduction du récit de fiction à la troisième personne au modèle du récit de fiction à la première personne, lui-même conçu sur le modèle du discours communicationnel. Elle retrouve au contraire la conception dualiste ou différentialiste traditionnelle, qui fait du récit de fiction à la première personne un cas particulier de récit de fiction, et lui apporte la caution de l'analyse linguistique ${ }^{6}$. Ensuite, cette théorie permet d'étudier le récit de fiction à la fois comme récit et comme fiction. Elle ne se débarrasse pas de la question de la fictionnalité en attribuant le récit à un narrateur fictionnel ou considéré comme tel, censé raconter des faits réels. Elle affirme au contraire que tous les récits de fiction ne sont pas racontés par un narrateur fictionnel et que, lorsqu'ils ne le sont pas, il n'y a aucune raison de recourir au même type d'analyse linguistique, narratologique ou reader-oriented que lorsqu'ils le sont (encore moins de leur substituer des analyses auxiliaires, introduites $a d$ $h o c$, c'est-à-dire dans l'unique intention de sauver une hypothèse menacée par des faits contraires ${ }^{7}$ ). Enfin, cette théorie est une théorie poétique de la narration, qui pose l'existence d'une intention poétique (de création), manifestable et reconnaissable, et qui ne se confond pas avec une intention de communication. Cela ne la condamne pas plus au subjectivisme que de postuler, par exemple, l'existence d'une intention de communication. En revanche, cela permet sa mise en relation avec d'autres théories poétiques, portant sur le roman, le théâtre ou la poésie.

1.2 Avant de tenter une approche de «Cinq ans de vie » étayée par la théorie de Kuroda et Banfield, je ferai quelques remarques au sujet des modalités d'application à l'étude de ce récit de certaines des propositions exposées précédemment.

«Cinq ans de vie » se présente comme un récit transfictionnel ${ }^{8}$, écrit à la troisième personne, avec représentation des pensées et états de conscience d'un personnage. Il se rapproche et se distingue à la fois du type de récit de fiction pour lequel la théorie de Kuroda et Banfield a été conçue (récit de fiction réaliste à la troisième personne, avec représentation des pensées et états de conscience d'un ou de plusieurs personnages). La narratologie, quant à elle, est doublement handicapée devant un tel récit, qui (1) n'est pas à la première personne (n'a ni narrateur ni narrataire explicites et contient des phrases qui excluent la présence d'un narrateur implicite ou effacé) et (2) présente des faits fictionnels sans correspondant dans le monde réel de référence, ce qui rend la supposition d'un narrateur fictionnel racontant des faits réels difficilement tenable. Du seul fait qu'elle s'exonère de ce double handicap, la théorie de Kuroda et Banfield apparaît comme plus prometteuse.

\subsubsection{Les faits fictionnels}

L'histoire se déroule à Paris à la fin des années 1960. Ses protagonistes sont Raúl Morales et Mirta Cisneros. Il est uruguayen, elle est argentine. Il est écrivain (il écrit des nouvelles), elle peint ou plutôt peignait avant d'arriver à Paris. Ils se rencontrent une nuit dans la station de métro Bonne Nouvelle, après la fermeture des portes de sortie. Ils parlent, ils se racontent leurs histoires, leurs espoirs déçus. À cinq heures moins le quart, Raúl dit à Mirta : «Tu sais quoi ? Je donnerais cinq ans de ma vie pour que tout commence ici. Je veux dire : 
j'aurais déjà divorcé, ma femme l'aurait accepté et ne se serait pas tuée, j'aurais trouvé un bon boulot à Paris et quand les portes s'ouvriraient, nous sortirions d'ici comme un couple. Ne le sommes-nous pas déjà ? ». Mirta répond : «Moi aussi je donnerais cinq ans » et ajoute : «Peu importe, on se débrouillera »" À cinq heures, les portes s'ouvrent, Raúl et Mirta sortent de la station et la suite des événements montre que les désirs exprimés par Raúl sur le mode de l'engagement à faire un grand sacrifice sont devenus réalité. Le dénouement de la nouvelle contient néanmoins une surprise sur laquelle je reviendrai.

Il s'agit donc d'un récit non réaliste ou transfictionnel. On a ici au moins deux transgressions de l'ordre établi dans le monde réel de référence : (1) la réalisation des désirs de Raúl, tous et tout de suite (ou le désir d'être transporté dans un futur conforme à ses désirs) ; (2) le fait que Raúl donne, c'est-à-dire sacrifie cinq ans de sa vie. Au moment où il sort de la station de métro, Raúl a divorcé, sa femme ne s'est pas tuée, il a trouvé un travail suffisamment stable et bien rémunéré pour pouvoir habiter un immeuble bourgeois, il est marié avec Mirta depuis plusieurs années : tout cela est donné comme réel et objectif dans le monde de la fiction. En même temps, Raúl a sacrifié cinq ans de sa vie, ce qui fait qu'il y a un manque, une lacune de cinq ans dans sa vie. Cela aussi, le lecteur doit l'accepter comme étant réel et objectif dans le monde de la fiction.

Je préfère parler de manque ou de lacune plutôt que d'ellipse, pour éviter les confusions. L'ellipse temporelle, telle qu'elle est définie par la narratologie, consiste dans l'élision dans le récit (la narratologie dit «par le narrateur ») d'un segment temporel de l'histoire, qui peut être décrit ou non dans la suite du récit, mais que le lecteur n'est pas autorisé à imaginer comme un segment manquant dans la vie des personnages ${ }^{10}$. La lacune de «Cinq ans de vie », elle, fait partie du monde de l'histoire, et le lecteur, une fois parvenu au terme du récit, est obligé de l'imaginer comme un segment manquant dans la vie de Raúl. J'ajoute qu'il n'y a aucune ellipse temporelle repérable dans le récit des événements qui constituent le dénouement de "Cinq ans de vie ».

\subsubsection{Le sujet de conscience}

«Cinq ans de vie » représente les pensées et états de conscience de Raúl et uniquement de lui (ce que la narratologie appelle une focalisation interne sur Raúl et une focalisation externe sur Mirta ${ }^{11}$ ). Contrairement à ce que présuppose la narratologie, ces passages ne sont pas le récit par un narrateur des événements intérieurs à la conscience de Raúl ; ils en sont la représentation directe, sans la médiation d'aucun point de vue (la troisième personne et le passé ne construisent pas ce que j'appelle ici une subjectivité ou un point de vue). Ils sont plus nombreux au début et à la fin de la nouvelle, c'est-à-dire de part et d'autre du récit de la nuit passée avec Mirta dans la station de métro, qui comporte en revanche de nombreux passages de discours direct.

1.3 Je limiterai mon étude au dénouement de la nouvelle, dans lequel la répartition de l'information narrative entre les phrases objectives et subjectives et les phrases du discours direct est particulièrement intéressante. Je prends dénouement dans un sens large, en y incluant à la fois le premier dénouement, qui s'avère être un faux dénouement (organisé autour de la révélation que les désirs de Raúl sont devenus réalité), et le dénouementsurprise. Mon étude portera sur le texte de la traduction française — un texte français, donc —, mais je ne m'interdirai pas de faire des comparaisons avec le texte espagnol, qui pourraient donner une justification indépendante de la théorie de Kuroda et Banfield. Je supposerai également que c'est dans le va-et-vient entre les deux langues que les spécificités du français apparaîtront de la façon la plus convaincante. Les deux versions, française et espagnole, du dénouement sont données à lire en annexe.

\section{Un dénouement sans parole}

\subsection{Raúl, Mirta et la « fille du Appleton » (ou le faux dénouement)}

2.1.1 Le premier paragraphe du dénouement est entièrement composé de phrases narratives ou objectives :

\footnotetext{
Le premier signe que la station reprenait son train-train quotidien fut un courant d'air. Ils éternuèrent tous les deux. Ensuite toutes les lumières s'allumèrent. Raúl lui tint son petit miroir pendant qu'elle se rendait présentable. Lui aussi mit un peu d'ordre dans ses cheveux. Alors qu'ils montaient lentement les escaliers, ils croisèrent la première avalanche de lève-tôt ${ }^{12}$.
}

Elles ont pour temps de base le passé simple en français et le pretérito indefinido en espagnol, qui a le même rôle que le passé simple lorsqu'il est combiné exclusivement à des formes de troisième personne et à des adverbes non déictiques (rôle masqué par le fait que le pretérito indefinido n'est pas exclu de l'appareil formel du discours, oral ou écrit). Elles établissent ce qui est de l'ordre des faits dans le monde de la fiction. On ne peut douter que le premier signe que la station reprend son train-train quotidien soit un courant d'air, ni que les personnages éternuent tous les deux, ni que toutes les lumières s'allument, ni non plus que ces faits se déroulent 
dans le monde fictionnel dans l'ordre dans lequel ils sont présentés dans le récit. La phrase suivante n’a pas un statut différent :

Il avançait en songeant qu'il ne l'avait même pas embrassée et se demandait s'il n'aurait pas dû être plus entreprenant.

Ce à quoi songe Raúl, ainsi que le fait qu'il songe et se demande telle ou telle chose, sont à considérer comme étant réels et objectifs dans le monde de la fiction. La dernière phrase du paragraphe pose un problème particulier :

Dehors il ne faisait pas aussi froid que la veille.

A priori, cette phrase est une phrase objective, en raison de l'expression non déictique «la veille» («la víspera »). On peut opposer, de ce point de vue, «Dehors il ne faisait pas aussi froid que la veille » et « Dehors il ne faisait pas aussi froid qu'hier ». Cependant, cette catégorisation n'est valable qu'en première lecture. En seconde lecture, si on connaît le dénouement de la nouvelle, on sait que, du point de vue référentiel, l'expression « la veille » est erronée, que « la veille » ne désigne pas la veille du jour où Raúl et Mirta sortent de la station, mais un jour ou plutôt une nuit cinq ans auparavant. Autrement dit, on est confronté au double paradoxe suivant. Si la phrase est une phrase objective, elle est fausse : donc, ce n'est pas une phrase objective. Si c'est une phrase subjective, elle est mal formée : donc, ce n'est pas une phrase (intentionnellement) subjective. Elle n'est pas faite pour que le lecteur de première lecture, qui est aussi le lecteur prévu par Benedetti au moment de l'écriture de la nouvelle, la prenne pour une phrase subjective, mais pour qu'il assume, sans s'en rendre compte, quelque chose d'erroné.

Je serai plus synthétique sur la suite de l'extrait, qui se compose essentiellement de phrases objectives et de phrases du discours direct, attribuées d'abord à Mirta («Et maintenant? » ${ }^{13}$ ), puis à la fille rencontrée sur le boulevard Bonne Nouvelle. Les premières rapportent un certain nombre de faits de manière objective : Raúl et Mirta vont ensemble vers une destination qu'ils ne connaissent pas ; ils rencontrent une fille qui les connaît, mais qu'eux ne connaissent pas $^{14}$; elle les entraîne vers la rue de l'Échiquier, puis vers un immeuble et un appartement qu'elle connaît, mais qu'eux ne connaissent pas. Les phrases du discours direct confirment le caractère réel et objectif de ces faits dans le monde de la fiction :

«Je vous trouve enfin, espèces de crétins! J'ai passé toute la nuit à vous appeler à l'appartement, et rien, aucune réponse. Où étiez-vous passés? J'ai besoin du Appleton de Raúl. Tu peux me le prêter? À moins qu'il ne soit à Mirta?»

Face à l'appartement numéro 7, la fille dit : «Bon, alors vous ouvrez ? »

«J'espère que vous aurez retrouvé la parole ce soir. Vous n'avez pas oublié qu'aujourd'hui on a décidé d'aller à la fête d'Emilia ? Apportez des disques. Please ! ${ }^{15}$

Ces faits n'ont qu'une explication possible dans le monde de la fiction. On peut la présenter de la façon suivante. Raúl et Mirta ont été transportés dans un autre monde (qu'on peut noter $\mathrm{M}_{2}$ ), homologue au monde des désirs de Raúl (ou du moins à une certaine description de ce monde, celle qui est contenue dans le passage : « Je donnerais cinq ans de ma vie », etc.). Dans le monde $\mathrm{M}_{2}$, Raúl et Mirta ont les propriétés de former un couple (le texte n'a pas encore dit qu'ils étaient mariés), de vivre ensemble dans le même appartement, d'avoir les mêmes amis et d'aller dans les mêmes fêtes. Mais les deux personnages ne le savent pas et croient qu'ils sont encore dans le monde $\mathrm{M}_{1}$, dans lequel ils viennent juste de se rencontrer. Le texte ne nomme pas l'opérateur d'exception (Dieu, le Diable, le destin...) qui a permis le passage de $\mathrm{M}_{1}$ à $\mathrm{M}_{2}$, qui se soustrait à l'intérêt du lecteur. Les mondes $\mathrm{M}_{1}$ et $\mathrm{M}_{2}$ font partie d'un espace-temps continu, comme en atteste par exemple :

D’un geste particulièrement prudent, Raúl décrocha de sa ceinture son vieux porte-clefs et vit qu'il y avait, comme toujours, trois clefs. 
La continuité est aussi indiquée, plus indirectement, par le «fort accent mexicain » de la fille et le prénom de l'organisatrice de la fête, Emilia ${ }^{16}$. Cependant, il s'agit bien de mondes différents, dans lesquels les propriétés assignées aux personnages sont différentes, comme en témoigne :

Il essaya la deuxième [clef] et parvint à ouvrir la porte.

On notera que certaines phrases objectives servent clairement à écarter la possibilité d'une explication réaliste (fondée sur l'idée d'un monde unique, celui que nous avons noté $\mathrm{M}_{1}$ ) :

Raúl pensa que ce devait être une amie de Mirta. Mirta pensa que c'était une copine de Raúl. ${ }^{17}$

On voit aussi à travers cet exemple que la logique du récit de Benedetti n'exclut pas de rapporter ou de raconter des pensées de Mirta dans le cadre de phrases objectives (bien qu'il n'y ait pas d'autre occurrence que celle-ci dans la nouvelle). Ce qu'elle exclut, c'est de les représenter dans des phrases ou des contextes subjectifs. Elle fait donc bien une différence entre les deux types de phrases.

On peut parler d'ironie narrative (équivalent, dans le domaine de la fiction narrative, de l'ironie dramatique au théâtre) dans la mesure où le lecteur comprend beaucoup plus vite que les personnages ce qui leur arrive - dès la fin du deuxième paragraphe, tandis que les personnages sont décrits comme cherchant à comprendre dans le troisième («chacun d'eux plongé dans ses propres pensées ») et, s'agissant de Raúl, comme n'ayant pas encore compris dans le quatrième.

2.1.2 Dans le quatrième paragraphe, le passage du passé simple à l'imparfait en français, du pretérito indefinido au pretérito imperfecto en espagnol, est l'indice du passage d'un contexte narratif ou objectif à un contexte où sont représentées les perceptions du sujet de conscience, Raúl :

\begin{abstract}
Raúl, sans prononcer un mot, les sourcils froncés et les yeux mi-clos, se mit à examiner l'appartement. Sur l'étagère il retrouva ses livres, marqués et annotés de son trait rouge caractéristique, mais il y en avait d'autres, nouveaux, aux pages à moitié coupées. Sur le mur du fond se trouvait sa chère reproduction de Miró ; mais il y en avait une aussi de Klee qu'il avait toujours convoitée. Sur la table il y avait trois photos : une de ses parents, une autre d'un monsieur ressemblant étonnamment à Mirta ; sur la troisième, Mirta et lui dans la neige, enlacés et apparemment très joyeux. ${ }^{18}$
\end{abstract}

On note aussi la présence d'éléments subjectifs enchâssables au sens de Banfield : l'adjectif évaluatif « chère » (« querida»), l'adverbe dérivé d'un adjectif également caractérisable comme évaluatif, "étonnamment» (« sospechosamente ») ${ }^{19}$. Ils indiquent que ces phrases représentent la conscience de Raúl, avec une certaine ambiguïté quant au niveau de conscience représenté (conscience réflexive ou conscience non réflexive). Selon l'analyse de Banfield, il suffirait d'ajouter un élément ou une construction non enchâssable, par exemple une question directe («Était-ce vraiment sa chère reproduction de Miró ? ») ou simplement un mot exclamatif («Oui, il l'avait toujours convoitée ») pour imposer l'interprétation qu'il s'agit de phrases représentant la conscience réflexive. On aurait alors affaire à la représentation des réflexions conscientes que se fait Raúl à propos de ce qu'il perçoit et non plus à la simple représentation de ses perceptions. Sans que cela contredise l'analyse linguistique de Banfield, il convient de faire remarquer que dans plusieurs de ces phrases ou fragments de phrase, les perceptions représentées comportent une dimension interprétative certaine, même si Raúl se trompe dans ses interprétations : ainsi lorsqu'il oppose «ses livres » à «d'autres, nouveaux » ou «sa chère reproduction de Miró » à celle de Klee. Cela ne contredit pas l'analyse linguistique de Banfield, mais cela marque peut-être une des limites de son apport à l'analyse et à l'interprétation littéraires.

L'ironie narrative culmine avec la périphrase « un monsieur ressemblant étonnamment à Mirta », pour désigner le père de Mirta.

Quant à la troisième photographie, celle de "Mirta et lui dans la neige, enlacés et apparemment très joyeux », elle remplit pour Raúl, à l'intérieur de la fiction, le même rôle que toute photographie dans la réalité, le rôle de témoin de «ce qui a été » («La Photographie ne dit pas (forcément) ce qui n'est plus, mais seulement et à coup 
sûr, ce qui a été $\left.»^{20}\right)$. Je cite encore Barthes, dont les propos cadrent parfaitement ici : "C'est une prophétie à l'envers : comme Cassandre, mais les yeux fixés sur le passé, elle ne ment jamais : ou plutôt, elle peut mentir sur le sens de la chose, étant par nature tendancieuse, jamais sur son existence. Impuissante aux idées générales (à la fiction), sa force est néanmoins supérieure à tout ce que peut, a pu concevoir l'esprit humain pour nous assurer de la réalité - mais aussi cette réalité n'est jamais qu'une contingence ("ainsi, sans plus") $)^{21}$.

2.1.3. L'analyse de ce passage nous permet déjà de conclure que la langue du récit de Benedetti ne présente aucun élément qui justifie le recours à une conscience autre que celle du sujet de conscience, Raúl. Les exemples donnés ne manifestent en rien la présence d'un narrateur. Dans les phrases de récit pur, les adverbes et les adjectifs qualificatifs comme «un peu» («un poco»), «lentement» (《lentamente»), 《particulièrement» («particularmente ), «petit» en français (l'espagnol utilise un suffixe diminutif), «présentable» («presentable $)$, «prudent» (« cauteloso »), «vieux» (« viejo ») ne sont pas des mots évaluatifs au sens de Banfield. Sur le plan sémantique, rien dans le texte n'indique la possibilité que Raúl ne mette pas un peu d'ordre dans ses cheveux, que les personnages ne montent pas lentement l'escalier, etc.; autrement dit, rien ne laisse penser que ces caractérisations sont le résultat d'une interprétation de la part d'un narrateur, la conséquence d'un savoir qu'il aurait ou l'expression d'une subjectivité qui serait la sienne, au lieu de renvoyer à des faits bien établis dans le monde de la fiction.

L'absence de narrateur permet aussi de comprendre les faits linguistiques du quatrième paragraphe, où il n'y a aucune place pour ses interventions ${ }^{22}$.

Certes, Benedetti aurait pu choisir la forme du récit de fiction à la première personne raconté par Raúl («Sur le mur du fond se trouvait ma chère reproduction de Miró "), etc.). Mais cela aurait entraîné certaines difficultés pour représenter sans ambiguïté la conscience de Raúl-personnage, au moment où il se livre à l'examen de l'appartement, par opposition à celle de Raúl-narrateur, racontant rétrospectivement. De manière générale, il aurait été très difficile d'évacuer toute problématique liée à la rétrospection et donc à la dissimulation d'informations cruciales.

L'interprétation de «la veille» dans «Dehors il ne faisait pas aussi froid que la veille» ne requiert pas le concept d'un narrateur fictionnel racontant des faits réels (donc faisant un mensonge ici), mais celui d'un auteur réel, sujet d'une stratégie textuelle telle qu'elle apparaît à partir du texte examiné (faisant, non pas un mensonge, mais un faux, une fausse phrase objective, dans notre terminologie).

\subsection{Raúl ou la reconnaissance (le dénouement-surprise)}

2.2.1 Il convient tout d'abord de remarquer que le dernier paragraphe introduit des perturbations dans le système des phrases du récit.

Depuis que la fille du Appleton avait fait irruption, il n'avait plus osé regarder Mirta en face. À présent oui, il la regarda. ${ }^{23}$

La deuxième phrase est au passé simple (au pretérito indefinido) et contient cependant deux éléments caractéristiques des phrases subjectives : l'adverbe déictique «à présent» («ahora ») et le mot exclamatif « oui »(« sí »). Dans la phrase suivante, elle aussi au passé simple :

Elle lui renvoya un regard sans ombres, un rien fatigué peut-être, mais serein.

la modalisation «peut-être » («tal vez ») indique clairement la possibilité que le regard de Mirta ne soit pas un rien fatigué ; autrement dit, elle indique que cette caractérisation est le résultat d'une interprétation de la part de Raúl. Enfin, dans :

Cela ne l'aida pas beaucoup ${ }^{24}$ pourtant, car, à cet instant précis, Raúl eut la certitude non seulement qu'il avait eu tort de divorcer de son épouse de Montevideo, une femme hystérique mais intelligente, râleuse mais le cœur sur la main, mais aussi que son deuxième mariage allait à vau-l'eau. 
tout se passe comme si Benedetti avait choisi d'articuler dans une seule phrase graphique plusieurs phrases différentes syntaxiquement et quant à leur statut objectif ou subjectif : "Raúl eut la certitude qu'il avait eu tort de divorcer de son épouse de Montevideo » (qu'on peut comparer à « Raúl pensa que ce devait être une amie de Mirta ») ; "C'était une femme hystérique mais intelligente, râleuse mais le cœur sur la main » ${ }^{25}$ (qu'on peut comparer à "Sur la table il y avait trois photos : une de ses parents, une autre d'un monsieur ressemblant étonnamment à Mirta [...]») — avec une ambiguïté pour la dernière : "Raúl eut la certitude que son deuxième mariage allait à vau-l'eau » ou « Son deuxième mariage allait à vau-l'eau ».

On notera que ces perturbations ou ces mélanges de régime sont presque exclusivement réservés à ce passage ${ }^{26}$, qui a un statut particulier puisqu'il précède immédiatement et prépare la surprise finale.

Le lecteur apprend en même temps que Raúl (en même temps que Raúl comprend ou, disons, réorganise le monde de ses croyances) le fait qu'il a divorcé de son épouse de Montevideo (introduit comme un présupposé), le fait qu'il le regrette, le fait ou le jugement subjectif que son deuxième mariage va à vau-l'eau. Il n'a plus ce « temps d'avance » sur Raúl qui caractérisait jusque là sa compréhension du dénouement. Il n’y a plus non plus de différence entre le fait et le jugement subjectif dans la présentation de l'information selon laquelle le deuxième mariage de Raúl va à vau-l'eau.

2.2.2 La nouvelle s'achève sur une phrase subjective :

Non pas qu'il n'aimât plus cette femme mince, frileuse, presque sans défense qui le regardait depuis le fauteuil en rotin, seulement c'était clair pour lui que dans ses sentiments actuels pour Mirta, il restait très peu de cet innocent, soudain, prodigieux, envahissant sentiment amoureux né cinq ans plus tôt, quand il l'avait connue en cette nuit incroyable, de plus en plus lointaine, de plus en plus floue, où, par un piège du hasard, ils étaient restés enfermés dans la station Bonne Nouvelle ${ }^{27}$.

Ce que Banfield écrit à propos des phrases de la conscience non réflexive est également valable pour cette phrase, qui représente la conscience réflexive ou la pensée. Elle n'est pas la « reproduction réaliste » de l'activité mentale ; elle ne crée pas un «esprit transparent» (sur le mode : le narrateur omniscient dit que Raúl a pensé : «Non pas que je n'aime plus cette femme mince», etc.). Le contenu de la conscience fait l'objet de reconstructions et de représentations hypothétiques, ou, pour mieux dire, est créé par ces constructions (les adjectifs de valorisation positive antéposés, par exemple).

La dernière phrase contient la surprise annoncée dans le titre du recueil, La muerte y otras sorpresas. Elle est créée par la révélation brutale d'une ironie de situation : les cinq ans que Raúl a sacrifiés en disant « Je donnerais cinq ans de ma vie pour que tout commence ici» sont les cinq ans qui le séparent, au moment de l'acte de conscience représenté dans la dernière phrase, de la nuit où il a rencontré Mirta («il restait très peu de cet innocent, soudain, prodigieux, envahissant sentiment amoureux né cinq ans plus tôt »); autrement dit, ce sont les cinq premières années de sa vie avec Mirta $^{28}$. La disparition de ces cinq ans implique comme corollaire un mariage que Raúl regrette et dont il ne garde d'autre souvenir heureux que celui de la lointaine rencontre dans la station de métro.

La dernière phrase révèle aussi une nouvelle facette de la personnalité de Raúl : son incapacité à aimer une femme pendant plus de cinq ans (il est clair que ce que Raúl ressent pour Mirta, malgré la dénégation initiale, ne peut plus s'appeler de l'amour - le texte espagnol utilise d'ailleurs deux mots plus différents qu'en français : «querer» et « enamoramiento $»^{29}$ ). Je ne crois pas que dans la construction de la nouvelle une voix se fasse entendre qui saurait la durée moyenne des amours, selon une vérité statistique à laquelle les amours singuliers ne se conforment pas toujours. Je pense plutôt que Benedetti attribue au personnage de Raúl une part de responsabilité dans son propre malheur.

Rétrospectivement, des phrases comme « Il avançait en songeant qu'il ne l'avait même pas embrassée » ou « sur la troisième [photographie], Mirta et lui dans la neige, enlacés et apparemment très joyeux » prennent une résonance pathétique qu'elles n'avaient pas à la première lecture.

\subsection{De la description à l'interprétation}

Il me semble avoir montré que la théorie des phrases sans parole de Kuroda et Banfield pouvait prétendre à l'adéquation descriptive dans le cas du dénouement de "Cinq ans de vie ». Mais l'intérêt d'une théorie se mesure aussi à ce qu'elle permet de construire des propositions interprétatives plus riches que celles auxquelles 
on accède par la simple lecture intuitive. La théorie des phrases sans parole satisfait-elle à cette condition ? C'est ce que je voudrais examiner maintenant en revenant sur quelques points précis ${ }^{30}$.

Je passe rapidement sur les phrases objectives. Elles sont indispensables à l'établissement de ce qui est de l'ordre des faits dans le monde de la fiction (elles excluent, par exemple, que ces faits ne soient que fantasmes, rêves ou hallucinations de la part des personnages). Elles sont soumises à un critère de cohérence. On peut ajouter qu'elles n'existent que dans le récit de fiction. Pour les personnages, à l'intérieur de la fiction, il n'y a pas de phrases objectives : le discours de la fille, par exemple, est étrange, il suscite l'interrogation («Ils restèrent muets et immobiles », «Ne restez pas comme ça, comme deux statues, pour ne pas dire comme deux idiots $»^{31}$ ), mais il n'a pas a priori de pouvoir d'authentification (on a vu, en revanche, que c'était le rôle de la photographie relativement à la réalité passée). Cette disparité est à l'origine de l'ironie narrative dont j'ai parlé.

Les phrases du discours direct établissent ce qui est de l'ordre des faits de discours dans le monde de la fiction (on ne peut douter que la fille dise ce que le texte lui fait dire), le discours lui-même étant à analyser comme relevant du domaine de la subjectivité. Dans l'ensemble des phrases du discours direct de « Cinq ans de vie », il convient d'isoler les phrases « Je donnerais cinq ans de ma vie pour que tout commence ici », etc. et « Moi aussi je donnerais cinq ans », auxquelles la nouvelle attribue le pouvoir exceptionnel d'influer sur le cours des événements. On peut d'ailleurs se demander si ce statut exceptionnel vient de ce qu'elles inscrivent les faits dans un monde possible, lequel est destiné à s'actualiser, ou de leur co-orientation parfaite, qui est cause que le monde possible en question va s'actualiser. Toujours est-il qu'un des effets de la nouvelle dans le monde du lecteur est de défamiliariser l'expression «Je donnerais cinq ans de ma vie » et peut-être, plus généralement, toute phrase au conditionnel exprimant l'irréel pur ou considéré comme tel.

S'agissant des phrases subjectives (autres que celles du discours direct), Benedetti exploite assez peu le fait qu'elles ne sont pas soumises au même critère de cohérence que les phrases objectives («[...] la phrase représentant la conscience peut très bien ne pas être cohérente avec les "faits" de l'histoire : un personnage peut fort bien se tromper, ne serait-ce que momentanément, c'est-à-dire dans son MAINTENANT ${ }^{32}$ ). Il ne l'exploite en fait que dans le quatrième paragraphe, consacré à l'examen de l'appartement (on l'a vu, Raúl se trompe en opposant «ses livres » à « d'autres nouveaux », les deux reproductions de Miró et de Klee, et en ne voyant qu'un monsieur ressemblant étonnamment à Mirta sur la troisième photo). Au contraire, les deux dernières phrases de la nouvelle, la phrase qui mélange les régimes objectif et subjectif et la phrase subjective finale, montrent une cohérence totale entre les faits établis dans le monde de la fiction et les faits vus à travers le prisme de la subjectivité de Raúl. Il y a un pathétique profond dans la manière dont Raúl prend conscience, sans aucune place pour le doute ou la possibilité de l'erreur, de ce qui constitue les faits dans le monde de la fiction.

Dans la théorie de Kuroda et Banfield, la phrase subjective représente le lieu où le sujet de conscience se substitue au locuteur, au sujet parlant. C'est aussi l'hypothèse interprétative que nous conduisent à échafauder les relations entre phrases objectives, phrases subjectives et phrases du discours direct. La dernière phrase du discours direct prêtée à l'un des deux protagonistes est : "Et maintenant? ». Un certain nombre de phrases objectives établissent ensuite le silence, l'absence de communication verbale et même de communication tout court entre les deux personnages : « Raúl sentit qu'elle lui avait ôté la question de la bouche. Mais il n'eut pas l'occasion de lui répondre »; «Ils restèrent muets et immobiles »; « Raúl et Mirta marchèrent derrière elle, sans se parler ni se toucher, chacun d'eux plongé dans ses propres pensées »; "Raúl, sans prononcer un mot, les sourcils froncés et les yeux mi-clos, se mit à examiner l'appartement »; "Depuis que la fille du Appleton avait fait irruption, il n'avait plus osé regarder Mirta en face ». Ces faits offrent un contraste frappant avec ceux de la nuit dans la station de métro : les personnages parlent beaucoup, échangent beaucoup, utilisent à plein la fonction communicative du langage. Il y a des phénomènes linguistiques très intéressants dans le texte espagnol, qui ne peuvent pas être rendus en français. "Mirá, dijo él, para que veas que soy comprensivo y poco exigente, voy a empezar yo » : dans cette phrase, Raúl utilise le tutoiement ordinaire (tuteo), comme il l'a proposé à Mirta peu de temps auparavant. Mais dans les phrases suivantes : "Cuando termine, si no te dormiste, decís vos tu cuento. Y conste que si te dormís, no me ofendo. ¿Trato hecho? ${ }^{33}$, il utilise le voseo pronominal et verbal, puis le voseo exclusivement verbal, soit des formes spécifiques aux pays du Río de la Plata. Façon indirecte, subliminale, de gagner la sympathie de Mirta, comme l'établit la suite du texte ${ }^{34}$. Un peu plus tard, Raúl réutilise le voseo pour obtenir des confidences : « ¿Vos tenés novio, o marido, o amigos? $»^{35}$.

Dans le dernier paragraphe de la nouvelle, il est clair que Raúl ne peut pas dire à Mirta qu'il ne l'aime plus ou que ses sentiments pour elle ne sont plus ceux qu'ils étaient cinq ans auparavant. On n'est même pas obligé de supposer que ses pensées prennent une forme verbale. Si c'était le cas, ce serait une forme très " écrite », très « littéraire », et on peut se rappeler que Raúl a, d'une certaine manière, renoncé à la littérature à la fin de la nuit passée avec Mirta («S'il avait été en train d'écrire une de ses belles petites nouvelles, bien différentes des romans de gare, il n'aurait pu se résoudre à écrire que cette fille était son destin. Heureusement il n'écrivait pas, il pensait seulement, aussi il put s'avouer sans aucun problème que cette jeune femme était son destin ${ }^{36}{ }^{36}$. Raúl, à la fin de la nouvelle, est une conscience séparée et solitaire, monadique dans la certitude qu'il a de son échec. 
Le fait que Benedetti ne représente pas les pensées de Mirta comme il le fait de celles de Raúl accentue le caractère mystérieux du personnage («Peu importe, on se débrouillera », "Elle lui renvoya un regard sans ombres, un rien fatigué peut-être, mais serein », « Cela ne l'aida pas beaucoup pourtant », « cette femme mince, frileuse, presque sans défense »). À la différence de Raúl, Mirta est caractérisée par une certaine forme de constance, appelée ici "sérénité » (dans une phrase qui mélange les régimes objectif et subjectif). Je pense qu'elle est valorisée par le texte. Mais je reconnais qu'on pourrait aussi interpréter l'attitude de Mirta comme un aveuglement sur les véritables sentiments de son mari.

On peut enfin remarquer, même s'il est toujours hasardeux de s'appuyer sur quelque chose qui n'est pas dans le texte, que la phrase subjective finale ne contient aucune inférence sur les pensées et états de conscience de Mirta, ni même aucune indication de ce que Raúl est conscient que Mirta est, elle aussi, un être de conscience. On peut peut-être rapprocher cela de sa conduite de fuite vis-à-vis de sa première femme («J'en ai assez de cette situation, mais je ne me décide pas à rompre. Quand je l'insinue dans mes lettres, elle m'écrit de longues tirades hystériques où elle menace de se tuer si je la quitte, et, bien sûr, je sais que c'est du chantage mais... et si elle se tuait? », " Tu sais quoi ? Je donnerais cinq ans de ma vie pour que tout commence ici. Je veux dire : j'aurais déjà divorcé, ma femme l'aurait accepté et ne se serait pas tuée »). En tout état de cause, la phrase subjective finale à la fois dit la faillite du couple et la montre formellement.

Au total, il me semble (1) que la théorie de Kuroda et Banfield nous fait percevoir des choses, des oppositions notamment, que nous n'aurions pas perçues autrement (l'opposition entre la communication et ce qui n'est pas elle, l'opposition entre ce qui est de l'ordre des faits fictionnels et ce qui relève du domaine de la subjectivité, ainsi que la disparition possible de cette opposition) ; (2) que ce que nous intuitionnons du sens du texte (autour des raisons de la faillite du couple formé par Raúl et Mirta), c'est la théorie qui nous permet de l'interpréter. La théorie est un vecteur d'interprétation.

Ce qui apparaît aussi dans la phrase finale, c'est que l'échec du personnage est contrebalancé par une réussite, qui est celle de la nouvelle elle-même ${ }^{37}$.

\section{Références bibliographiques}

Arregui, M., éd. (1968). La otra mitad del amor contada por siete hombres. Montevideo : Editorial Arca.

Banfield, A. (1991). L'écriture et le non-dit. Diacritics, 21.4, 21-31.

- (1995 [1982]), Phrases sans parole. Théorie du récit et du style indirect libre. Traduit de l'anglais par Cyril Veken. Paris : Le Seuil.

Barthes, R. (1980). La Chambre claire. Note sur la photographie. Paris : Éditions de l'Étoile, Gallimard, Le Seuil.

Benedetti, M. (1968). Cinco años de vida. In La muerte y otras sorpresas. Buenos Aires : Editorial Sudamericana, 122-133.

Benedetti, M. (1982 [1960]). La Trêve. Traduit de l'espagnol par Annie Morvan. Paris : Belfond, rééd. 1996.

Benedetti, M. (2009 [1968]). Cinq ans de vie. In Histoires de Paris. Traduit de l'espagnol (Uruguay) par Anne Casterman. Noville-sur-Mehaigne (Belgique) : Esperluète Éditions, 17-32.

Berthelot F. (2010). Narratologie thématique et narratologie discursive : le cas des transfictions ». In John Pier et Francis Berthelot, éds., Narratologies contemporaines. Approches nouvelles pour la théorie et l'analyse du récit. Paris, Éditions des archives contemporaines, 75-89.

Genette, G. (2007 [1972]). Discours du récit. Paris, Le Seuil, rééd. «Points ».

Kuroda, S.-Y. (1979 [1973]). Où l'épistémologie, la grammaire et le style se rencontrent : examen d'un exemple japonais. Trad. de l'anglais par Cassian Braconnier. In Aux quatre coins de la linguistique. Paris: Le Seuil, "Travaux linguistiques $», 235-259$.

Kuroda, S.-Y. (à paraître, 2012). Pour une théorie poétique de la narration. Traduit de l'anglais par Cassian Braconnier, Tiên Fauconnier et Sylvie Patron. Paris : Armand Colin, « Recherche».

Patron, S. (2009). Le Narrateur. Introduction à la théorie narrative. Paris : Armand Colin, «U».

Patron, S. (2011). La mort du narrateur et l'interprétation du roman. L'exemple de Pedro Páramo de Juan Rulfo. In Sylvie Patron, éd., Théorie, analyse, interprétation des récits / Theory, analysis, interpretation of narratives. Berne : Peter Lang, 147-182.

Patron, S. (à paraitre 2012). Les récits de fiction antérieurs à la deuxième moitié du XIX ${ }^{\mathrm{e}}$ siècle : des instances de réfutation pour les théories poétiques de la narration ? Le Français moderne, 1, Récits parlés, récits montrés : l'hypothèse non communicationnelle du récit de fiction à l'épreuve des textes d'Ancien Régime, 15-31. 
Rama, Á., éd. (1966). Aquí la mitad del amor contada por seis mujeres. Montevideo : Editorial Arca.

Ryan, M.-L. (2010). Cosmologie du récit: des mondes possibles aux univers parallèles. In Françoise Lavocat, éd., $L a$ Théorie littéraire des mondes possibles. Paris : CNRS Éditions, 53-81. 


\title{
Annexes
}

\author{
Mario Benedetti, "Cinq ans de vie ", Histoires de Paris, traduit de l'espagnol (Uruguay) par \\ Anne Casterman, Noville-sur-Mehaigne (Belgique), Esperluète Éditions, 2009, p. 30-32
}

Le premier signe que la station reprenait son train-train quotidien fut un courant d'air. Ils éternuèrent tous les deux. Ensuite toutes les lumières s'allumèrent. Raúl lui tint son petit miroir pendant qu'elle se rendait présentable. Lui aussi mit un peu d'ordre dans ses cheveux. Alors qu'ils montaient lentement les escaliers, ils croisèrent la première avalanche de lève-tôt. Il avançait en songeant qu'il ne l'avait même pas embrassée et se demandait s'il n'aurait pas dû être plus entreprenant. Dehors il ne faisait pas aussi froid que la veille.

Sans se consulter, ils empruntèrent le boulevard Bonne Nouvelle, en direction du bureau de poste. «Et maintenant ? », dit Mirta. Raúl sentit qu'elle lui avait ôté la question de la bouche. Mais il n'eut pas l'occasion de lui répondre. Depuis le trottoir d'en face, une fille, en pantalon noir et pull vert, leur faisait de grands signes pour qu'ils l'attendent. Raúl pensa que ce devait être une amie de Mirta. Mirta pensa que c'était une copine de Raúl. Finalement la fille parvint à traverser la rue et les interpella, pleine d'énergie, avec un fort accent mexicain : «Je vous trouve enfin, espèces de crétins ! J'ai passé toute la nuit à vous appeler à l'appartement, et rien, aucune réponse. Où étiez-vous passés ? J'ai besoin du Appleton de Raúl. Tu peux me le prêter ? À moins qu'il ne soit à Mirta ? »

Ils restèrent muets et immobiles. Mais l'autre renchérit: «Allez, ne soyez pas vaches ! J'en ai vraiment besoin. On m'a commandé une traduction. Super, non? Ne restez pas comme ça, comme deux statues, pour ne pas dire comme deux idiots. Vous allez à l'appartement ? Je vous accompagne. » Elle prit la rue Mazagran en direction de la rue de l'Échiquier, ponctuant sa démarche pressée d'un mouvement des hanches bien rythmé. Raúl et Mirta marchèrent derrière elle, sans se parler ni se toucher, chacun d'eux plongé dans ses propres pensées. La fille tourna le coin et s'arrêta devant le numéro 28. Ils montèrent tous les trois l'escalier (il n'y avait pas d'ascenseur) jusqu'au quatrième étage. Face à l'appartement numéro 7, la fille dit: "Bon, alors vous ouvrez? » D'un geste particulièrement prudent, Raúl décrocha de sa ceinture son vieux porte-clefs et vit qu'il y avait, comme toujours, trois clefs. Il essaya la première sans succès. Il essaya la deuxième et parvint à ouvrir la porte. La fille se rua sur l'étagère de livres qui se trouvait près de la fenêtre, elle arracha presque l'Appleton, embrassa Raúl sur les deux joues, ensuite Mirta et dit : «J'espère que vous aurez retrouvé la parole ce soir. Vous n'avez pas oublié qu'aujourd'hui on a décidé d'aller à la fête d'Emilia? Apportez des disques. Please ! » Et sur ce, elle sortit comme une flèche en claquant la porte.

Mirta se laissa tomber dans le fauteuil en rotin. Raúl, sans prononcer un mot, les sourcils froncés et les yeux mi-clos, se mit à examiner l'appartement. Sur l'étagère il retrouva ses livres, marqués et annotés de son trait rouge caractéristique, mais il y en avait d'autres, nouveaux, aux pages à moitié coupées. Sur le mur du fond se trouvait sa chère reproduction de Miró ; mais il y en avait une aussi de Klee qu'il avait toujours convoitée. Sur la table il y avait trois photos : une de ses parents, une autre d'un monsieur ressemblant étonnamment à Mirta; sur la troisième, Mirta et lui dans la neige, enlacés et apparemment très joyeux.

Depuis que la fille du Appleton avait fait irruption, il n'avait plus osé regarder Mirta en face. À présent oui, il la regarda. Elle lui renvoya un regard sans ombres, un rien fatigué peut-être, mais serein. Cela ne l'aida pas beaucoup pourtant, car, à cet instant précis, Raúl eut la certitude non seulement qu'il avait eu tort de divorcer de son épouse de Montevideo, une femme hystérique mais intelligente, râleuse mais le cœur sur la main, mais aussi que son deuxième mariage allait à vau-l'eau. Non pas qu'il n'aimât plus cette femme mince, frileuse, presque sans défense qui le regardait depuis le fauteuil en rotin, seulement c'était clair pour lui que dans ses sentiments actuels pour Mirta, il restait très peu de cet innocent, soudain, prodigieux, envahissant sentiment amoureux né cinq ans plus tôt, quand il l'avait connue en cette nuit incroyable, de plus en plus lointaine, de plus en plus floue, où, par un piège du hasard, ils étaient restés enfermés dans la station Bonne Nouvelle. 


\begin{abstract}
Mario Benedetti, "Cinco años de vida ", La muerte y otras sorpresas, Buenos Aires, Editorial sudamericana, 1968, p. 130-133 [en ligne] <http://lyc5.files.wordpress.com/2011/06/mariobenedetti-la-muerte-y-otras-sorpresas.pdf> (consulté le 7 novembre 2011)
\end{abstract}

El primer síntoma de que la estación reanudaba su rutina, fue una corriente de aire. Ambos estornudaron. Luego se encendieron todas las luces. Raúl sostuvo el espejito mientras ella se ponía presentable. Él mismo se peinó un poco. Cuando subían lentamente las escaleras, se cruzaron con la primera avalancha de madrugadores. Él iba pensando en que ni siquiera la había besado y se preguntaba si no se habría pasado de discreto. Afuera no hacía tanto frío como la víspera.

Sin consultas previas, empezaron a caminar por el boulevard Bonne Nouvelle, en dirección a la sucursal de Correos. ¿Y ahora?, dijo Mirta. Raúl sintió que le había quitado la pregunta de los labios. Pero no tuvo oportunidad de responder. Desde la acera de enfrente, otra muchacha, de pantalones negros y buzo verde, les hacía señas para que la esperaran. Raúl pensó que sería una amiga de Mirta. Mirta pensó que sería una conocida de Raúl. Al fin la chica pudo cruzar y los abordó con gran dinamismo y acento mexicano: Al fin los encuentro, cretinos. Toda la noche llamándolos al apartamento, y nada. ¿Dónde se habían metido? Necesito que Raúl me preste el Appleton. ¿Puedes? ¿O acaso es de Mirta?

Quedaron mudos e inmóviles. Pero la otra arremetió. Vamos, no sean malos. De veras lo preciso. Me encargaron una traducción. ¿Qué les parece? No se queden así, como dos estatuas, por no decir como dos idiotas. ¿Van al apartamento? Los acompaño. Y arrancó por Mazagran hacia la rue de l'Echiquier, acompañando su apuro con un bien acompasado movimiento de trasero. Raúl y Mirta caminaron tras ella, sin hablarse ni tocarse, cada uno metido en su propia expectativa. La chica nueva dobló la esquina y se detuvo frente al número 28. Los tres subieron por la escalera (no había ascensor) hasta el cuarto piso. Frente al apartamento 7, la muchacha dijo: Bueno, abran. Con un movimiento particularmente cauteloso, Raúl descolgó del cinto su viejo llavero, y vio que había, como siempre, tres llaves. Probó con la primera; no funcionó. Probó con la segunda y pudo abrir la puerta. La chica atropelló hacia el estante de libros que estaba junto a la ventana, casi arrebató el Appleton, besó en ambas mejillas a Raúl, luego a Mirta, y dijo: Espero que cuando venga esta noche hayan recuperado el habla. ¿Se acuerdan de que hoy quedamos en ir a lo de Emilia? Lleven discos, please. Y salió disparada, dando un portazo.

Mirta se dejó caer sobre el sillón de esterilla. Raúl, sin pronunciar palabra, con el ceño fruncido y los ojos entornados, comenzó a revisar el apartamento. En el estante encontró sus libros, señalados y anotados con su inconfundible trazo rojo; pero había otros nuevos, con las hojas a medio abrir. En la pared del fondo estaba su querida reproducción de Miró; pero además había una de Klee que siempre había codiciado. Sobre la mesa había tres fotos: una, de sus padres; otra, de un señor sospechosamente parecido a Mirta; en la tercera estaban Mirta y él, abrazados sobre la nieve, al parecer muy divertidos.

Desde que apareciera la chica del Appleton, no se había atrevido a mirar de frente a Mirta. Ahora sí la miró. Ella retribuyó su interés con una mirada sin sombras, un poco fatigada tal vez, pero serena. No la ayudó mucho, sin embargo, ya que en ese instante Raúl tuvo la certeza, no sólo de que había hecho mal en divorciarse de su esposa montevideana, histérica pero inteligente, malhumorada pero buena hembra, sino también de que su segundo matrimonio empezaba a deteriorarse. No se trataba de que ya no quisiera a esa delgada, friolenta, casi indefensa mujer que lo miraba desde el sillón de esterilla, pero para él estaba claro que en sus actuales sentimientos hacia Mirta quedaba muy poco del ingenuo, repentino, prodigioso, invasor enamoramiento de cinco años atrás, cuando la había conocido en cierta noche increíble, cada vez más lejana, cada vez más borrosa, en que, por una trampa del azar, quedaron encerrados en la estación Bonne Nouvelle. 
* Une première version de cet article est parue dans Littérature au présent. Mélanges offerts à Francis Marmande, textes réunis par Sylvie Patron, Textuel, $\mathrm{n}^{\circ}$ 67, 2012.

${ }^{1}$ Histoires de Paris, traduit de l'espagnol (Uruguay) par Anne Casterman, Noville-sur-Mehaigne (Belgique), Esperluète Éditions, 2009, p. 17-32. Les nouvelles qui composent ce recueil sont issues de trois ouvrages antérieurs de Mario Benedetti. "Cinco años de vida » a été publiée pour la première fois dans La muerte y otras sorpresas (voir Benedetti, 1968). Elle a été republiée la même année dans une anthologie de nouvelles éditée par Mario Arregui sous le titre La otra mitad del amor contada por siete hombres, qui fait pendant à une autre anthologie de nouvelles, Aquí la mitad del amor contada por seis mujeres (Rama, éd., 1966).

${ }^{2}$ Voir Kuroda, à paraître, 2012 ; Banfield, 1995 [1982]. Voir aussi Patron, 2009, dont la deuxième partie est consacrée aux théories poétiques de la narration.

${ }^{3}$ Banfield, $1991: 27$ (je traduis).

${ }^{4}$ Kuroda, 1979 [1973] : 256, repris dans Kuroda, à paraître, 2012 (je remplace « récits écrits dans le style rapporté », qui prend sens à l'intérieur du système théorique de Kuroda, par « récits à la première personne », qui renvoie à la plus importante sous-catégorie de « récits écrits dans le style rapporté »).

${ }^{5}$ Banfield, 1995 [1982] : 313-314 (traduction légèrement modifiée).

${ }^{6}$ Voir Patron, 2009 : 12-16 et passim, et à paraître, 2012.

7 Voir l'analyse de Marie-Laure Ryan dans «Cosmologie du récit: des mondes possibles aux univers parallèles » : «Je veux bien faire semblant de croire à un univers où Emma Bovary existe, se suicide, a les pensées que rapporte le narrateur. Mais je n'imagine pas que dans ce monde les pensées sont transparentes (la preuve, c'est que Charles est incapable de lire les pensées d'Emma !) ; et je n'imagine pas non plus qu'il existe un seul individu, le narrateur, qui a le don de lire les pensées. Dans mon acte d'imagination, je mets l'existence du narrateur entre parenthèses » (Ryan, $2010: 58$, n. 7).

${ }^{8}$ J'emprunte ce terme à Francis Berthelot dans « Narratologie thématique et narratologie discursive : le cas des transfictions » (voir Berthelot, 2010). Berthelot désigne ainsi les fictions (romans et nouvelles) situées dans la zone frontalière entre littérature générale et littératures de l'imaginaire (science-fiction), et caractérisées par une transgression ou de l'ordre du monde ou des lois du récit.

9 « Cinq ans de vie », p. 30.

${ }^{10}$ Voir Genette, 2007 : 103-106.

${ }^{11}$ Voir ibid. : 197.

${ }^{12}$ « Cinq ans de vie », p. 30.

${ }^{13}$ Ibid., p. 31.

${ }^{14}$ La phrase «Depuis le trottoir d'en face, une fille, en pantalon noir et pull vert, leur faisait de grands signes pour qu'ils l'attendent» est ambiguë quant à son statut objectif ou subjectif (possibilité d'adjonction d'un adverbe déictique comme «maintenant»; absence effective de tout élément lexical ou grammatical à fonctionnement déictique ; absence également d'éléments lexicaux ou grammaticaux non déictiques).

${ }^{15}$ Dans le texte espagnol, « lo de Emilia », traduit ici par « la fête d'Emilia », est une expression du langage familier.

${ }^{16}$ Voir le début de la nouvelle où Raúl est obligé de partir d'une réunion amicale chez des Boliviens pour ne pas rater le dernier métro ; voir aussi p. 22 : «Il se promettait toujours de nouer une relation plus ou moins stable avec une Française - rien de tel pour s'imprégner définitivement de la langue - mais au bout du compte ses amitiés tant féminines que masculines se limitaient au clan latino-américain ».

${ }^{17}$ Dans le texte espagnol, le parallélisme entre les deux phrases est plus marqué. D’autre part, « conocida » n’a pas la connotation familière de « copine ».

${ }^{18}$ «Cinq ans de vie », p. 32. 
${ }^{19}$ Sur les éléments subjectifs enchâssables, voir Banfield, 1995 [1982] : 150-152. Sur les adjectifs évaluatifs, voir ibid.: 100-103 (le critère définitoire des adjectifs évaluatifs est qu'ils peuvent apparaître dans une exclamative du type adjectif + nom; exemple : «Pauvre garçon!»).

${ }^{20}$ Barthes, $1980: 133$.

${ }^{21}$ Ibid. : 134-135.

${ }^{22}$ Voir Banfield, 1995 [1982] : 293.

${ }^{23}$ «Cinq ans de vie», p. 32.

${ }^{24}$ Le texte espagnol dit : « No la ayudó mucho » (« Cela ne l'aida pas beaucoup, elle »).

${ }^{25}$ L'adjectif « hystérique » est repris du discours direct de Raúl s'adressant à Mirta dans la station de métro (voir p. 28 : «J'en ai assez de cette situation, mais je ne me décide pas à rompre. Quand je l'insinue dans mes lettres, elle m'écrit de longues tirades hystériques où elle menace de se tuer si je la quitte, et, bien sûr, je sais que c'est du chantage, mais... et si elle se tuait ? »).

${ }^{26}$ On peut également citer p. 30 : «Après cela, il poussa un soupir ; on pouvait l'interpréter comme un premier soupir prometteur » («Después de eso, suspiró ; podía ser interpretado como un suspiro de inauguración », p. 130) et, dans le texte espagnol, p. 127 : «Trato hecho, dijo ella, sonriendo francamente y tendiéndole, ahora sí, la mano » (voir infra n. 34), ainsi que deux occurrences de la combinaison pretérito indefinido + "próxima » ( «prochaine »). Cependant, le statut déictique de cet adjectif n'est pas aussi évident en espagnol qu'en français. Il est d'ailleurs traduit par « suivante », qui est son correspondant non déictique.

${ }^{27}$ Dans le texte espagnol, la dernière proposition est au pretérito indefinido, qui s'emploie beaucoup plus couramment que le passé simple pour exprimer l'antériorité et qui se traduit fréquemment par le plus-que-parfait. On peut aussi noter l'usage du déterminant démonstratif «esa » dans «esa mujer », qui peut avoir une connotation péjorative, impossible à rendre en français.

${ }^{28}$ On peut rapprocher la mésaventure de Raúl de ce passage de La Trêve (La tregua, 1960), roman réaliste et roman écrit en forme de journal : «Je sais qu'il ne me reste que quatre mois de factures, de contrats, de bilans partiels, de comptes courant, de déclarations... mais je donnerais une année de ma vie pour que ces quatre mois se réduisent à zéro. Enfin, en y réfléchissant un peu, je ne donnerais rien du tout parce que ma vie, maintenant, c'est Avellanada [nom de famille du personnage féminin] » (Benedetti, $1996: 159)$. On passe de l'un à l'autre par un double processus d'expansion et de littéralisation.

${ }^{29}$ Voir aussi la description de l'« énamoration » p. 30 : «L'émotion qui suivit fut quelque chose de plus qu'un simple état d'âme ; ce fut réellement une exaltation organique qui envahit ses oreilles sa gorge, ses poumons, son cœur, son estomac, son sexe, ses genoux » (p. 130 : «La emoción subsiguiente fue algo más que un estado de ánimo ; realmente fue una exaltación orgánica que abarcó orejas, garganta, pulmones, corazón, estómago, sexo, rodillas »).

${ }^{30}$ En cela, cet article fait pendant à «La mort du narrateur et l'interprétation du roman. L'exemple de Pedro Páramo de Juan Rulfo » (Patron, 2011), où je m'intéressais surtout aux problèmes d'interprétation soulevés par la théorie et l'analyse narratologiques, opposées à la théorie poétique de la narration.

${ }^{31}$ « Cinq ans de vie », p. 31.

${ }^{32}$ Banfield, 1995 [1982] : 322. «MAINTENANT » désigne le point de référence à partir duquel sont interprétés les adverbes déictiques de temps qui peuvent apparaitre dans les phrases subjectives.

33 «Cinco años de vida», p. 127 («Cinq ans de vie», p. 26 : «Écoute, dit-il, c'est moi qui vais commencer comme ça tu verras que je suis tolérant et peu exigeant. Quand je terminerai, si tu ne t'es pas endormie, ce sera à ton tour de me raconter ton histoire. Et rassure-toi, si tu t'endors, je ne me vexerai pas. Marché conclu ?»).

${ }^{34}$ Voir ibid. : « Il sentit que, grâce à cette dernière proposition, il avait adroitement gagné sa sympathie. "Marché conclu", dit-elle avec un franc sourire en lui tendant enfin la main ».

35 «Cinco años de vida», p. 129 (« Cinq ans de vie», p. 28 : «Et toi tu as un fiancé, ou un mari, ou des amis? »).

${ }^{36}$ «Cinq ans de vie », p. 30. Dans le texte espagnol, c'est le même mot, «muchacha », qui est utilisé dans les deux phrases (traduit ici par « fille» et « jeune femme »). Il contraste avec le mot « mujer » (« femme ») qui est utilisé dans la dernière phrase de la nouvelle. 
${ }^{37}$ Dans la traduction française, l'effet de clôture est plus marqué en raison du jeu de mots possible sur le nom de la station : «Bonne Nouvelle». 